\title{
MUI-Suppression with Reduced T-Equalizer for UTRA FDD Downlink
}

\author{
Klaus Knoche \\ OSB AG \\ Klenzestr. 38 \\ D-80469 Munich, Germany \\ Email: k.knoche@osb-ag.de
}

\author{
Wen $\mathrm{Xu}$ \\ Siemens AG - Mobile Phones \\ Grillparzerstr. 10 - 18 \\ D-81675 Munich, Germany \\ Email: Wen.Xu@siemens.com
}

\author{
Jürgen Rinas and Karl-Dirk Kammeyer \\ Department of Communications Engineering \\ University of Bremen \\ Otto-Hahn-Allee, D-28359 Bremen, Germany \\ Email: \{rinas, kammeyer\}@ ant.uni-bremen.de
}

\begin{abstract}
Wideband CDMA systems with orthogonal spreading codes suffer severely due to the loss of orthogonality by multi-path propagation. This yields Multiple User Interference (MUI), which gravely reduces the performance of classical systems with Rake-receivers. In our approach we attempt to restore orthogonality by using a modified T-equalizer. Classical T-equalizers are composed as FIR filters with equidistant delays and appropriate coefficients. Their main disadvantage is the high computational effort due to the large number of coefficients.

The question arises whether we need to calculate all equally spaced coefficients, or if some of the coefficients can be neglected. The basic idea of this paper is to use only a subset of coefficients and set all others to zero. The main advantages are the reduced computational effort in calculating the filter and the decreased computational costs in using it. In this paper we will show the feasibility with some simulation results for block fading channels. In practice, we usually do not use a T-equalizer very often for time variant channels, due to its high computational costs. An adaptive algorithm is instead taken and modified accordingly. In our case we take a Least Mean Squares (LMS) algorithm for MUI-suppression in a time variant environment. In order to show the feasibility of this approach, some simulation results for channels with low Doppler frequencies are presented and compared with the classical Rake-receiver and a full version of the LMS/Griffith by [1].
\end{abstract}

\section{INTRODUCTION}

One feature of UMTS is the orthogonality of its spreading codes, the so called Orthogonal Variable Spreading Factor (OVSF) codes. This property gets lost on the way between base station and mobile station due to the multi-path propagation. One possibility to fight intra-cell Multi User Interference (MUI), is to re-establish the orthogonality of these codes by using a zero-forcing approach or by trying to find a compromise between noise enhancement and orthogonality with a Wiener filter scheme [2].

This is in fact also our approach, namely by using a classical tapped delay line equalizer. The T-equalizer is a FIR filter with equidistant (chip-rate) taps of significant length. In the case of a classical T-equalizer the computational effort is proportional to the number of coefficients to the power of three. For each less coefficient we save one complex multiplication per chip in actual operation. The question arises whether we need to calculate all of the coefficients, or if there are coefficients which are more important than others. We are not going to calculate a large number of equidistant coefficients but

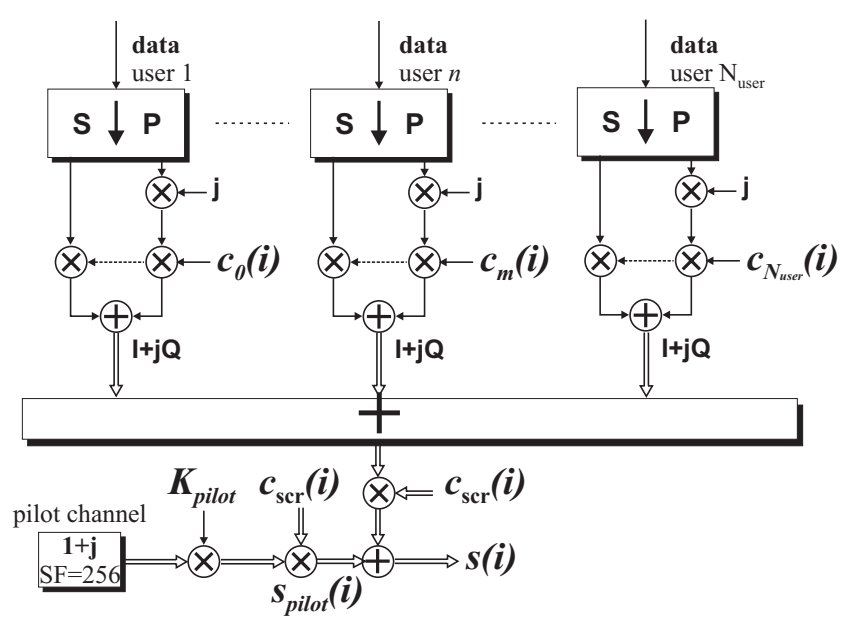

Fig. 1. Simplified transmitter scheme

only a small subset and force all the other coefficients to be zero. Hence we need only a fraction of computational costs at the price of a minor degradation. This is the main idea of this paper. Due to the reduced number of coefficients, the equalizer becomes numerically very stable which is an additional benefit.

In this paper we will show the feasibility of this approach with some simulations for block fading channels.

Since the direct approach is quite expensive in terms of computational effort, many approaches use iterative algorithms like Recursive Least Squares (RLS) or LMS to adapt its coefficients to the time variant channel. A typical example based on this idea is the approach proposed in [1] for adapting a T-equalizer to the time variant channel using an LMS algorithm which is used for reference. The LMS algorithm can also be used for our modified version of the T-equalizer and some simulation results will be presented for slow time variant channel.

\section{System Model}

We have taken a CDMA system and consider the whole system as a chip rate model. Upon others UMTS defines an FDD wideband CDMA scheme. Here only the downlink is considered. The spreading is done with help of an OVSF 
(Walsh) code with a spreading factor $G_{S F}$ between 4 and 512 and an additional scrambling with a truncated complex valued Gold code with the length of one frame equal to 38400 chips or $10 \mathrm{~ms}$. In our case the scrambling code is the same for every user in one cell. In general, a QPSK modulation is used in downlink direction ${ }^{1}$. The data $d_{n}(k)$ of the $\mathrm{n}$-th user with index $k$ denoting the symbol rate is mapped to chip rate by

$$
\tilde{d}_{n}(i)=\sum_{k}\left(d_{n}(k) \cdot \sum_{l=0}^{G_{S F}-1} \delta\left(i-l-k \cdot G_{S F}\right)\right)
$$

The index $i$ denotes the chip rate. After this the signal is spread with its user dependent OVSF-code denoted with $c_{n}(i)$. The resulting signal at the transmitter output of the base station is the sum of all users multiplied with the scrambling code $c_{\mathrm{Scr}}(i)$ plus an additional pilot channel $s_{\text {pilot }}(i)$. In our case we assume that all users have the same spreading factor $G_{S F}$ and the same power.

$$
s(i)=\left(\sum_{n=1}^{N_{\text {user }}}\left(\tilde{d}_{n}(i) \cdot c_{n}(i)\right)\right) \cdot c_{\mathrm{Scr}}(i)+s_{\text {pilot }}(i)
$$

The pilot channel has a fixed spreading factor of 256, contrary to the ones used for the data. Its OVSF-code is a simple repetition code $(256 \times 1)$. The power of the pilot channel is $10 \%$ of the total transmit power of the considered cell and is represented by the amplification factor $K_{\text {pilot }}$. This simplified transmitter in respect to the UMTS standard, is depicted in Fig. 1.

The output signal of the transmitter is convolved with the mostly time varying channel impulse response $h(i, \rho)$ and distorted by a zero-mean additive white Gaussian noise (AWGN).

$$
r(i)=s(i) * h(i, \rho)+n(i)
$$

The classical receiver structure is the Rake-algorithm which is the best possible single user detector in case that all distortions can be assumed as white Gaussian noise. In cases where the distortions are not Gaussian, such as in most practical situations, the G-Rake algorithm can be utilized in the receiver (see [3]). Another typical scheme for solving this problem, as known from non-CDMA systems, is the use of an equalizer.

\section{T-EQUALIZER FOR CDMA SYSTEMS}

If we use a normal FIR-filter with its coefficients e for equalizing the channel influence, the resulting output signal $x(i)$ of the equalizer is

$$
x(i)=\hat{s}(i)=e(i) * r(i) .
$$

By correlating the signal $x(i)$ with the channelization (OVSF) code and the scrambling code, we receive an estimate for

\footnotetext{
${ }^{1}$ We omit the special cases like the High Speed Downlink Packet Access (HSDPA).
}

the data $\hat{d}(k)$ of the $n$-th user. The classical solution for the coefficients e is to solve the Eq. ( 5 [4])

$$
\mathbf{e}_{M M S E}=\left(\mathbf{H}^{H} \mathbf{H}+\gamma \mathbf{I}\right)^{-1} \mathbf{H}^{H} \mathbf{i}
$$

with $\mathbf{H}$ denoting the convolution matrix of the channel coefficients $\mathbf{h}, \mathbf{I}$ is the identity matrix. The factor $\gamma$ can be seen as a compromise between noise magnification and remaining MUI. For reasons of simplicity we assume that each signal for every user is broadcast with the same power. In this case the number of users has to be taken into account. For the classical MMSE-filter in the single user narrow-band environment $\gamma$ is normally set to

$$
\gamma^{2}=\frac{N_{0}}{E_{S}}
$$

In this case the system normally deals with the effects of ISI of one user. In a spread system with a single user $^{2} \frac{N_{0}}{E_{S}}$ will change to $\frac{N_{0}}{E_{c}}$ with $E_{c}$ denoting the energy per chip and $G_{S F}$ denoting the spreading factor. This yields

$$
\gamma^{2}=\frac{N_{0}}{E_{s}} \cdot \frac{G_{S F}}{N_{u s e r}} .
$$

The variable $N_{\text {user }}$ denotes the number of user for the special case that all users have the same power.

\section{REDUCED T-EQUALIZER}

The equalizer described above used an equidistant chip spacing. Instead of using all coefficients, we try to reduce the number of coefficients and neglect the equidistant condition. In the following example we set the coefficient $e_{1}$ to zero.

$$
\underbrace{\left(\begin{array}{llll}
h_{0} & \emptyset & 0 & 0 \\
h_{1} & h_{0} & 0 & 0 \\
h_{2} & h_{1} & h_{0} & 0 \\
0 & h_{2} & h_{1} & h_{0} \\
0 & \emptyset & h_{2} & h_{1} \\
0 & \varnothing & 0 & h_{2}
\end{array}\right)}_{\mathbf{H}} \cdot\left(\begin{array}{l}
e_{0} \\
e_{1} \\
e_{2} \\
e_{3}
\end{array}\right)=\left(\begin{array}{l}
0 \\
0 \\
1 \\
0 \\
0 \\
0
\end{array}\right)+\delta
$$

This results in the following equation:

$$
\left(\begin{array}{lll}
h_{0} & 0 & 0 \\
h_{1} & 0 & 0 \\
h_{2} & h_{0} & 0 \\
0 & h_{1} & h_{0} \\
0 & h_{2} & h_{1} \\
0 & 0 & h_{2}
\end{array}\right) \cdot\left(\begin{array}{l}
e_{0} \\
e_{2} \\
e_{3}
\end{array}\right)=\left(\begin{array}{l}
0 \\
0 \\
1 \\
0 \\
0 \\
0
\end{array}\right)+\delta
$$

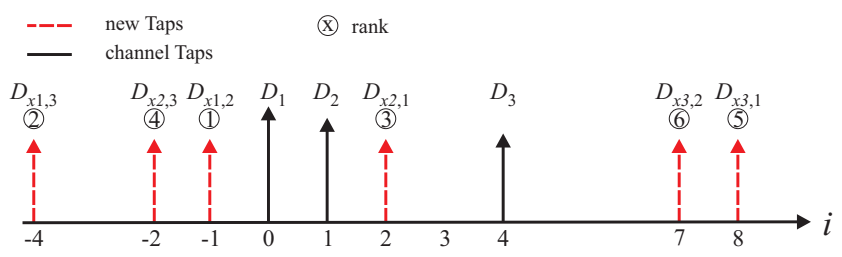

Fig. 2. Selection routine according to [5]

${ }^{2}$ Of course we would prefer the MRC-receiver in this case. 


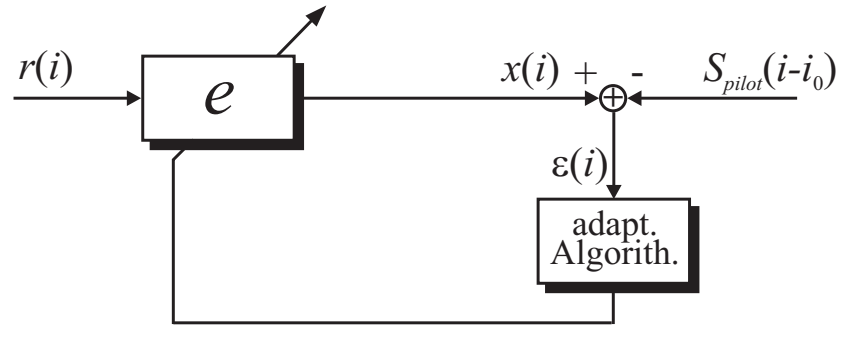

Fig. 3. LMS adaption scheme

In order to solve it we take again Eq. ( 5) analogue to the normal case. The problem left is which coefficients to take and which ones to neglect. At first we take all coefficients according to the channel impulse response. Additionally we are looking for some extra coefficients. In [5] an algorithm was presented for choosing the appropriate positions for additional coefficients for the G-Rake by [3], which can also be applied to our problem and is depicted in Fig. 2. The algorithm by [5] searches for the strongest channel coefficient, takes the distance to the second strongest and puts a new finger on the opposite site. Then it compares the position of the strongest channel coefficient with the third strongest and so on. The position of the second strongest channel coefficient is taken as compared with the strongest, the third strongest etc. till all other fingers are assigned.

This can be easily done due to the similarity of this reduced T-equalizer and the G-Rake by [3]. Although the approaches are different, both algorithms need to calculate the inverse of an autocorrelation matrix. In our case the autocorrelation matrix of the incoming signal $\mathbf{R}_{\mathbf{r r}}{ }^{-1}$ needs to be computed, and in the other one for a matched filter for colored noise the autocorrelation matrix of the distortion $\mathbf{R}_{\mathbf{u u}}{ }^{-1}$. At the input of our CDMA-receiver on the other hand, we receive a signal whose autocorrelation matrix consists mostly of distortions by white Gaussian noise and the other users, thus we have

$$
\mathbf{R}_{\mathbf{r r}}{ }^{-1} \approx \mathbf{R}_{\mathbf{u u}}{ }^{-1}
$$

\section{REDUCED LMS-ALGORITHM}

Eq. (4) can be rewritten according to [4], [6] for zero-mean signals at the receiver input.

$$
x(i)=\hat{s}(i)=\mathbf{h}^{H} \mathbf{R}_{\mathbf{r r}}{ }^{-1} \mathbf{r}=\mathbf{e}^{T} \cdot \mathbf{r} .
$$

with $\mathbf{R}_{\mathbf{r r}}$ denoting the autocorrelation matrix of the received signal $\mathbf{r}$. In order to avoid the expensive inversion of the autocorrelation matrix, as done in the previous section, the well-known LMS-algorithm can be used.

$$
\mathbf{e}^{T}(i+1)=\mathbf{e}^{T}(i)-\mu \cdot \epsilon(i) \cdot \mathbf{r}^{H}
$$

with

$$
\epsilon(i)=\mathbf{e}^{T} \cdot \mathbf{r}-s_{\text {pilot }}\left(i-i_{0}\right)=x(i)-s_{\text {pilot }}\left(i-i_{0}\right)
$$

For each filter coefficient we want to use, Eq. ( 12) yields:

$$
e_{\nu}(i+1)=e_{\nu}(i)-\mu \cdot \epsilon(i) \cdot r^{*}(i-\nu)
$$

This LMS-algorithm needs a reference which can be taken from the Common Pilot Channel (CPICH) for example. For further investigations we assume perfect channel knowledge and use the Griffith approach instead of a reference. This approach is done by calculating the expected value of the filter coefficients in Eq. ( 12). Instead of the reference $s_{\text {pilot }}(i)$ we need the cross correlation between the received and the transmitted signal which are the channel coefficients in conjugated and reversed order respectively.

$$
\begin{aligned}
E\left\{\mathbf{e}^{T}(i+1)\right\} & =\mathbf{e}^{T}(i)-\mu \cdot E\left\{x(i) \cdot \mathbf{r}^{H}\right\} \\
& +\mu \cdot E\left\{s_{\text {pilot }}\left(i-i_{0}\right) \cdot \mathbf{r}^{H}\right\} \\
\mathbf{e}^{T}(i+1) & =\mathbf{e}^{T}(i)-\mu \cdot\left(x(i) \mathbf{r}^{H}+\mathbf{h}_{-}^{H}\right)
\end{aligned}
$$

But instead of calculating all equidistant coefficients we again take only a subset and use the algorithm presented in [5].

\section{Simulation Results}

In the following section we present some simulation results for block fading channels. Both channels used, are defined in [7].

TABLE I

3GPP CHANNEL MODELS ACCORDING TO [7]

\begin{tabular}{|l|ccccc|}
\hline Taps/Chips: & 0 & 1 & 2 & 3 & 4 \\
Rel. Delay: & $0 \mathrm{~ns}$ & $260 \mathrm{~ns}$ & $521 \mathrm{~ns}$ & $781 \mathrm{~ns}$ & $976 \mathrm{~ns}$ \\
\hline Case 3: & $0 \mathrm{~dB}$ & $-3 \mathrm{~dB}$ & $-6 \mathrm{~dB}$ & $-9 \mathrm{~dB}$ & - \\
Case 4: & $0 \mathrm{~dB}$ & - & - & - & $0 \mathrm{~dB}$ \\
\hline
\end{tabular}

In Fig. 4, the simulation results for a 1/4, 1/2 loaded system for the case 3 channel are presented. For this 4-tap channel we used a Rake-receiver, a 19-tap equidistant chip-wise Tequalizer and a 6-tap reduced T-equalizer and an AWGN curve for QPSK as reference. The spreading factor was set to $G_{S F}=$ 128 and no channel coding was applied. The pilot channel was omitted. At a BER of $10^{-2}$ the 6-tap modified equalizer loses $0.4 \mathrm{~dB}$ and $1.5 \mathrm{~dB}$ for the $1 / 4$ and $1 / 2$ loaded system, respectively, compared to the T-equalizer with 19 taps. The gain compared to the Rake-receiver is about $4 \mathrm{~dB}$.

In Fig. 5 the simulation results for a 1/4, 1/2 loaded system for the case 4 channel are presented. Because this is actually a 2-path Rayleigh channel, we additionally added the appropriate theoretical curve [4]. Instead of taking a 19 tap Tequalizer we are now using a 21 tap version and the reduced T-equalizer with 4 taps. The reason for T-equalizer of order 20 is to make sure that we have more than 4 usable taps. Due to the structure of the channel only every fourth tap differs from zero. Therefore with only 19 taps and centered orientation we get exact the same results for the T-equalizer as for the 4 tap reduced version. At a BER of $10^{-2}$ the 4 tap modified equalizer loses nearly nothing, and $0.5 \mathrm{~dB}$ for the $1 / 4$ and $1 / 2$ loaded system in respect to the equispaced T-equalizer ${ }^{3}$, and gains $1.25 \mathrm{~dB}$ or more for the $1 / 4$ and $1 / 2$ loaded system compared to the Rake-receiver.

\footnotetext{
${ }^{3}$ The gain of about $0.1 \mathrm{~dB}$ is due to simulation inaccuracies.
} 


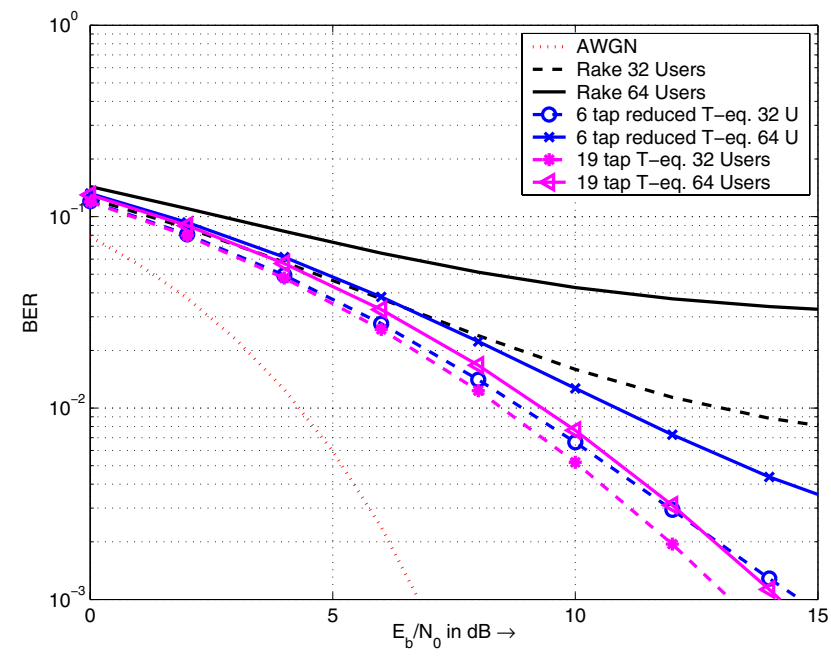

Fig. 4. Simulation results for Case 3 channel and $G_{S F}=128$, uncoded

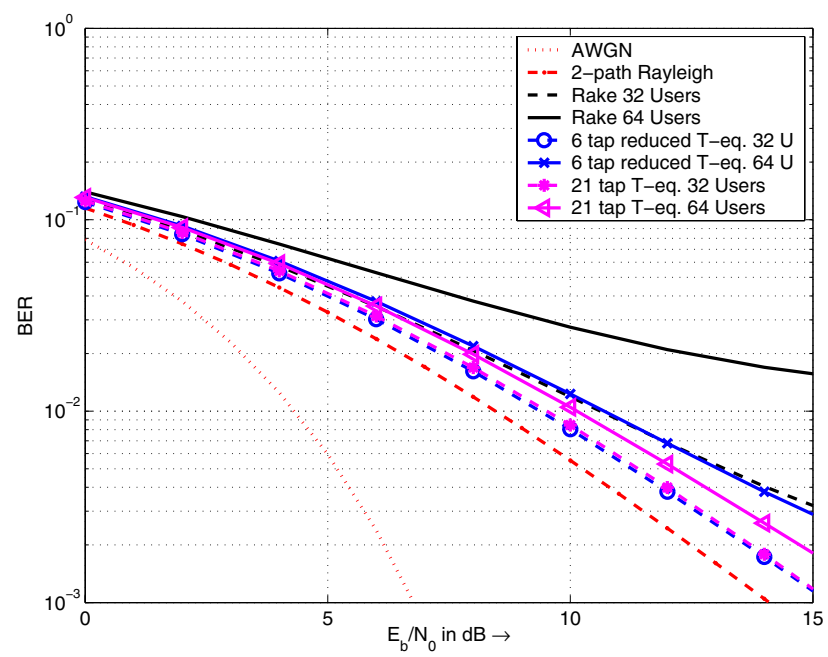

Fig. 5. Simulation results for Case 4 channel and $G_{S F}=128$, uncoded

In both cases we only added two more fingers compared to the Rake-receiver and obtained a nice gain compared to the Rake-receiver. The performance loss is acceptable even compared to the classical T-equalizer with 19 or 21 taps, which can be seen as a 19 or 21 finger Rake-receiver, respectively.

In Fig. 6, a 3GPP case 4 channel was taken and a velocity of $3 \mathrm{~km} / \mathrm{h}$ was assumed. We took again a four coefficients FIR-filter for the reduced version and a 21 tap FIR-filter as reference but took the LMS/Griffith algorithm for adaptation. The step factor $\mu$ was set to 0.0001 for both algorithms. We also assume perfect channel knowledge, that we utilize with the Griffith approach. Because the slow time variant channel is easy to adapt, the loss in performance can be neglected. Consequently, the results are quite similar compared to the block fading case of the T-equalizer and the reduced Tequalizer respectively. A higher degradation is expected for the case of a proper channel estimation or for higher velocities ${ }^{4}$.

\footnotetext{
${ }^{4}$ even without taking the weaker CE into account
}

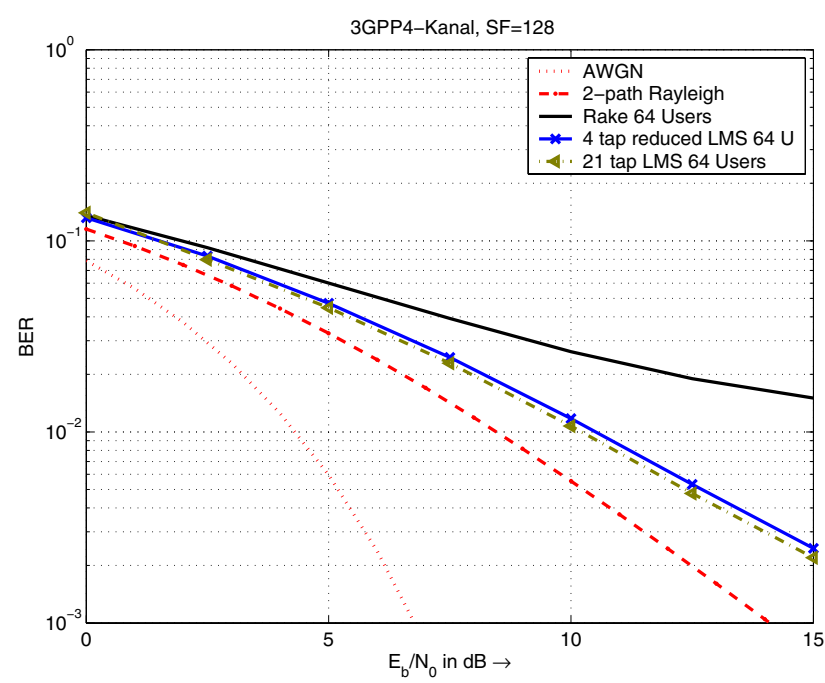

Fig. 6. Simulation results for Case 4 channel, half loaded system and $G_{S F}=$ 128 , uncoded

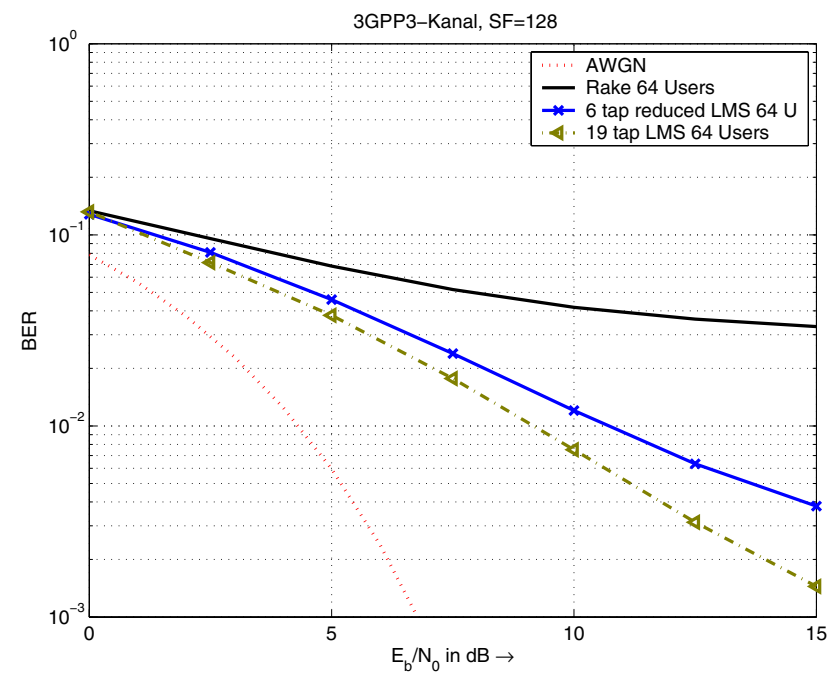

Fig. 7. Simulation results for Case 3 channel half loaded system and $G_{S F}=$ 128 , uncoded

In these cases the use of a normal LMS, with the CPICH as reference would be practicable and the step factor needs to be optimized.

In Fig. 7, a 3GPP case 3 channel was taken and the reduced LMS algorithm got 6 taps and the normal version has 19 taps. The results are mostly alike compared to the block fading case. Note that for this case the reduced LMS is just a normal equispaced equalizer designed with reduced order.

\section{CONCLUSION}

In this paper an idea to reduce the computational complexity for CDMA System with T-equalizers was presented. The point was to use only these coefficients that are important and neglect the rest. As a result the computational cost was decreased significantly for the T-equalizer while the performance degradation was quite small for the taken 3GPP channels. It 
was also presented that this idea can also be applied in an adaptive algorithm like the LMS. The algorithm for choosing the right coefficients was borrowed from [5], which was initially used for the finger placement of the G-Rake algorithm.

Since a limited number of channel models were studied here, investigating other channels and the application to other adaptation algorithms like RLS is certainly an interesting future task. Investigations on the degradation in the presence of a non-perfect channel estimation, including the use of the $\mathrm{CPICH}$ within the LMS algorithm, remains another interesting topic for further study.

\section{REFERENCES}

[1] M. J. Heikkilä, "A Novel Blind Adaptive Algorithm for Channel Equalization in WCDMA downlink," in Proc. IEEE Int. Symposium on Personal, Indoor and Mobile Radio Commun. (PIMRC), 2001.

[2] C.D. Frank, E. Visotsky, and U. Madhow, "Adaptive Interference Suppression for Downlink of a Direct Sequence CDMA System with Long Spreading Sequences," 1998.
[3] G.E. Bottomley, T. Ottosson, and Y.-P. E. Wang, "A Generalized RAKE Receiver for Interference Surpression," IEEE Journal on Selected Areas in Communications, vol. 18, no. 9, pp. 1536-1545, August 2000.

[4] K.-D. Kammeyer, Nachrichtenübertragung, Teubner, Stuttgart, second edition, 1996.

[5] G. Kutz and A. Chass, "On the Performance of a Practical Downlink CDMA Generalized RAKE Receiver," in IEEE Semiannual Vehicular Technology Conference (VTC) Fall 2002, Vancouver, Canada, October 2003.

[6] S. Kay, Fundamentals of Statistical Signal Processing, Estimation Theory, Prentice Hall International, London, first edition, 1993.

[7] 3rd Generation Partnership Project, TS 25.101 UE Radio transmission and Reception FDD, June 2001.

[8] J. G. Proakis, Digital Communications, McGraw-Hill, third edition, 1995.

[9] S. Haykin, Adaptive Filter Theory, Prentice Hall International, London, third edition, 1996

[10] K.-D. Kammeyer and K. Kroschel, Digitale Signalverarbeitung: Filterung und Spektralanalyse mit MATLAB Übungen, Teubner, Stuttgart, fourth edition, 1998. 